\title{
APPLICATION OF SATELLITE ALTIMETER DATA TO ORBIT ERROR CORRECTION AND GRAVITY MODEL ADJUSTMENT
}

\author{
R. C. A. Zandbergen, K. F. Wakker, B. A. C. Ambrosius \\ Delft University of Technology, Faculty of Aerospace Engineering, Kluyverweg \\ 1, 2629 HS Delft, The Netherlands
}

\begin{abstract}
The full exploitation of the oceanographical information contained in altimeter height observations made during altimetry missions of the past (SEASAT), present (GEOSAT) and future (ERS-1, TOPEX/Poseidon), requires very accurate satellite orbits. Although the dynamic models involved in the orbit computation are of steadily increasing accuracy, the strived-for accuracy of $10 \mathrm{~cm}$ of the radial position component may not be achieved for all these missions in the near future.

Several techniques have been devised to apply altimeter or cross-over data to satellite orbit correction and gravity model adjustment. This paper will address two methods which are currently being developed at Delft University of Technology (DUT). Using analytical theory, a gravity model may be adjusted with a limited number of laser range and cross-over difference observations by applying constraint matrices. This technique was successfully applied to tune the GEM-T1 model. With the same theory a new method of orbit error correction used by the so-called cross-over minimization technique for restricted areas has been devised, which allows the recovery of the eliminated orbit error, and the combination of these errors from several areas into a global error model.
\end{abstract}

\section{INTRODUCTION}

Since its launch in 1978 the SEASAT altimetry satellite has been the subject of orbit computation with steadily increasing accuracy. In succession to the final SEASAT-tailored gravity model called PGS-S4 it is currently the general-purpose model GEM-T1 which provides the most accurate orbits, with a radial accuracy of $50 \mathrm{~cm}$ rms. The key to the improved accuracy of the orbit was indeed the improved gravity field model, and though this is not the only source for the remaining orbit error, it is still the most important one. Because the accuracy of the radial component of the computed orbit should be $10 \mathrm{~cm}$ or less in order to apply the altimeter observations with their full precision to ocean surface modeling, a further improvement of the gravity field model is required.

For the TOPEX/Poseidon mission, which is to fly in the early 1990's, this improvement is already taking place on a grand scale, and the projected radial accuracy for the orbit of this satellite is some $15 \mathrm{~cm}$. It has to be mentioned here that this satellite will be using a higher orbit than SEASAT, with a completely different inclination. Another altimetry mission planned for the same timespan is the European ERS-1. This satellite will be flying an orbit much more similar to SEASAT, but because of the difference in orbital inclination, the radial error of ERS-1 orbits computed with current gravity models is estimated at $1 \mathrm{~m}$ or more.

Delft University of Technology's Section Orbital Mechanics (DUT/SOM) has been involved in SEASAT precise orbit determination and altimeter data processing for a number of years, and is 
currently preparing for its part in both the ERS-1 and TOPEX/Poseidon projects. The emphasis for DUT/SOM in these studies will lie on ERS-1 orbit improvement, preferably to the same standard as aimed for in the TOPEX/Poseidon mission, so that the altimeter data of these satellites may be merged to form one global set of equal precision. The contribution to the TOPEX/Poseidon mission will be that accurate altimeter data become available at those extreme latitudes that the TOPEX/Poseidon satellite will not reach.

A very useful tool in the experiments in the preparatory phase of this study is the SEASAT satellite. The first subject in this paper is a gravity model tuning experiment, which is a test case for a future tailoring of a general-purpose model to ERS-1. In this experiment the model was actually tailored to SEASAT, which is considered to be sufficiently similar to ERS-1 for a meaningful interpretation of the results. First of all the state-of-the-art accuracy of SEASAT orbits is established. After some general remarks about estimating the accuracy of gravity models, a general theory is outlined with which the subset of the gravity coefficients to be adjusted is defined. Then follows a description of the model adjustment itself and the results, and a discussion of how this may contribute to the ERS-1 mission.

The final subject in this paper is a presentation of a new non-dynamical orbit error correction procedure for regional applications. This procedure resembles the traditional tilt-and-bias local crossover minimization scheme. It has, however, a number of advantages, which will be discussed in the pertinent sections.

\section{ACCURACY ASSESSMENT OF SEASAT ORBITS}

The accuracy of SEASAT orbit computation has been established by many authors over the last few years [1,2,3], and the values most cited in literature are the $\mathrm{ms}$ radial position error of $70 \mathrm{~cm}$ for PGS-S4 orbits and $50 \mathrm{~cm}$ for GEM-T1 orbits. Tables 1 and 2 show some recent results by DUT/SOM for four 3-day SEASAT arcs in the period of september 15 to 271978 . These tables list the statistics of the laser range residuals and altimeter cross-over difference residuals, from which an estimate of the global radial orbit error is deduced. In both cases the orbits were computed using Geodyn.

In the first case (Table 1) the PGS-S4 gravity model, the GEM-10B-detailed geoid model [4] and the PGS-S4 station coordinates were applied. This combination will be referred to as the 'PGS-S4 model'. The 'GEM-T1 model' (Table 2) represents the combination: preliminary GEM-T1 gravity field, GEM-10B-detailed geoid and UT/CSR 8402 station coordinates. More detailed information about the applied models and tracking data may be found in [3].

The radial orbit error estimates in Tables 1 and 2 were computed as:

(1) $1 / 2 \sqrt{2}$ times the cross-over residual rms.

(2) The low-frequency part of the altimeter residuals $(f<1.5 \mathrm{cpr})$.

(3) Combination of model (1) and an estimate of the geographically correlated error.

This correlated error was computed as a combination of four Legendre functions of latitude and longitude, which were fitted through the altimeter residuals, and their $\mathrm{ms}$ about mean is also listed in Tables 1 and 2. In the ideal case estimates (2) and (3) should have similar values. The low-frequency part of the altimeter residuals (estimate (2)) should not contain contributions other than the radial orbit error, while orbit errors with higher frequency which are contained in estimate (3) through the cross-over residual rms can only be very small. The occurring discrepancy, observed in Tables 1 and 2, has been thoroughly investigated. In experiments it was found that the discrepancy would almost completely disappear if the GEM-10B-detailed geoid were replaced by a GEM-10B or a PGS.S4 geoid. This leads to the conclusion that estimate (3) is the most reliable one, and that estimate (2) is influenced by the relatively high noise level of the detailed geoid. Another feature discovered in the high-frequency part of the altimeter residuals was a latiude-dependent signal with a frequency of 2 cycles per orbital revolution, equivalent to a flattening effect. The amplirude of this signal of approximately $1.1 \mathrm{~m}$ varied depending on the applied geoid model. The major part of this 
signal is probably due to an equatorial bulge in the oceans due to ocean dynamics features [5].

The final estimate ((3)) of the SEASAT radial orbit accuracy, of $90 \mathrm{~cm}$ and $60 \mathrm{~cm}$ for orbits, computed with the PGS-S4 and GEM-T1 models respectively, are somewhat higher than the values mentioned above, but this is mainly due to the geographical correlation of the radial orbit error for the PGS-S4 orbits, which has been identified as the result of a common $z$-shift of $1 \mathrm{~m}$ in the PGS-S4 station coordinates [3]. This may be demonstrated by Figure 1, which shows the geographically correlated part of the radial orbit error mentioned in Tables 1 and 2 and also that of the difference between the two orbits for one of the arcs. The geographically correlated error appears as a latitudedependent error in the PGS-S4 orbit. The radial difference between the two orbits is also represented in the frequency domain in Figure 2, which shows that there is a significant peek near one cycle per revolution, and that the difference gradually decreases for high frequencies. It may be assumed that the error for each of the gravity models is similar to this figure, because both models are representative for SEASAT orbit computation.

Because the major part of the remaining radial orbit error is due to errors in the gravity field model, extensive research and development of gravity field modeling is currently being undertaken by various groups, of which the NASA GSFC GEM-T1 model is one of the first major results [6]. Until now DUT/SOM has concentrated its efforts in this area towards the tuning of existing gravity models to specific satellites. Some preliminary results in this field have already been published $[7,8]$, whereas this paper addresses a new development in gravity model tuning, showing that SEASAT laser and cross-over residuals may be significantly reduced with respect to those for GEM-T1 orbits, by adjusting a subset of the GEM-T1 gravity model coefficients.

Table 1. Cross-over difference residuals and estimated radial orbit error statistics for four SEASAT arcs computed with the 'PGS-S4 model', no ocean tides applied. All values are in $\mathrm{cm}$. The radial orbit error statistics were estimated as follows: 1) $1 / 2 \sqrt{2}$ times the ms cross-over residuals. 2) Rms about mean of the low-frequency part of altimeter residuals. 3) Combination of 1) and the 4 Legendre functions.

\begin{tabular}{lccccr}
\hline Arc & Xover & Legendre & \multicolumn{3}{c}{ Rms radial orbit error } \\
ID & rms & functions & model1 & model2 & model3 \\
\hline C2 & 100 & 65 & 71 & 130 & 96 \\
C3 & 90 & 64 & 64 & 120 & 90 \\
C4 & 125 & 78 & 88 & 137 & 118 \\
C5 & 85 & 58 & 59 & 112 & 83 \\
\hline
\end{tabular}

Table 2. Cross-over difference residuals and estimated radial orbit error statistics for four SEASAT arcs computed with the 'GEM-TI model', including ocean tides. All values are in $\mathrm{cm}$.

\begin{tabular}{cccccc}
\hline Arc & Xover & Legendre & \multicolumn{3}{c}{ Rms radial orbit ertor } \\
ID & rms & functions & model1 & model2 & model3 \\
\hline C2 & 77 & 21 & 54 & 106 & 58 \\
C3 & 71 & 20 & 50 & 100 & 54 \\
C4 & 74 & 21 & 52 & 101 & 56 \\
C5 & 82 & 19 & 58 & 101 & 61 \\
\hline
\end{tabular}



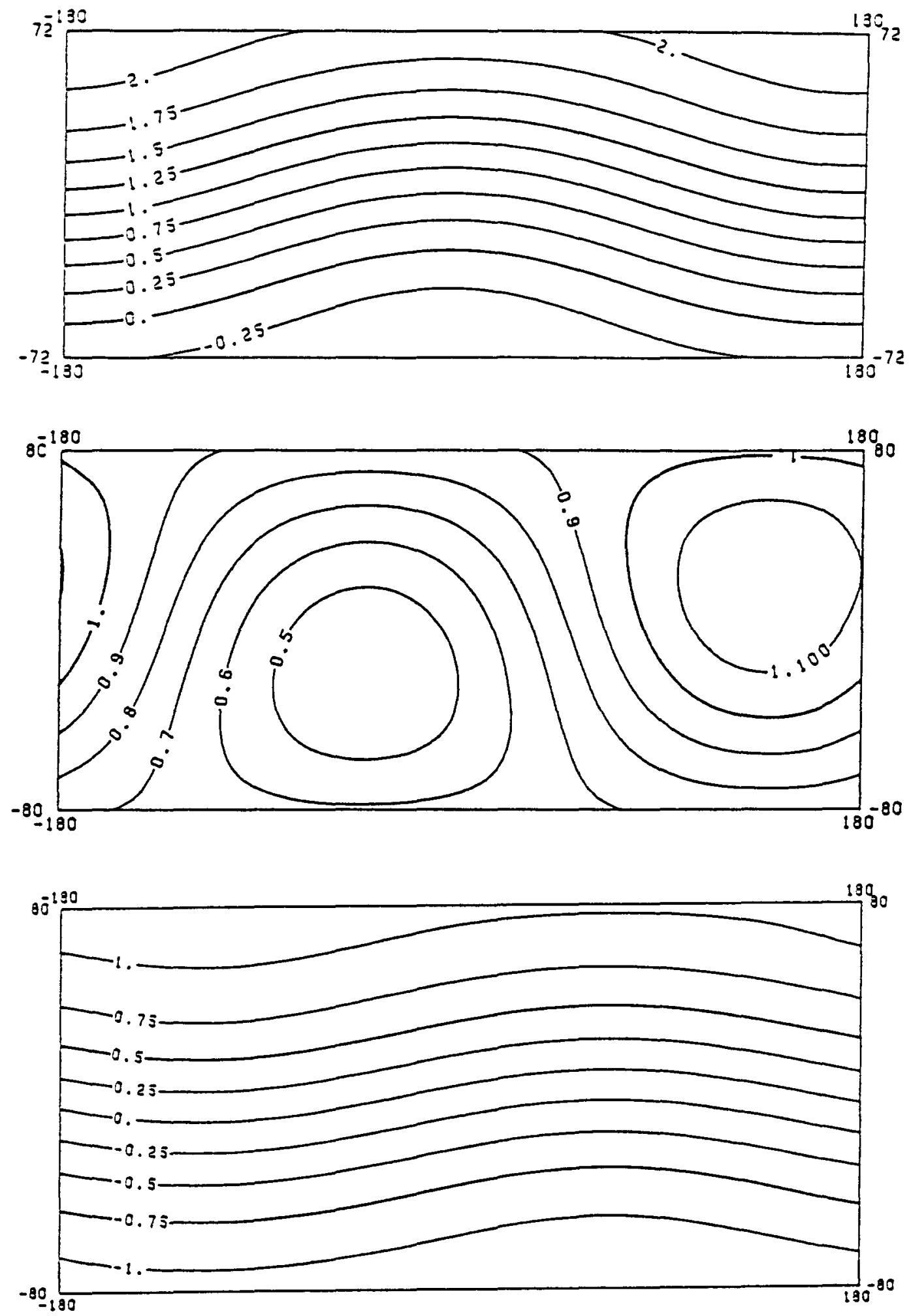

Figure 1. The geographically correlated radial orbit error plotted as a function of latitude and longitude. In all three cases the error was computed as a combination of the four Legendre functions of degrees 0 and 1. TOP: The radial orbit error for the 'PGS-S4 model', estimated from altimeter residuals. CENTER: The radial orbit error for the 'GEM-T1 model' estimated from altimeter residuals. BOTTOM: The correlated part of the radial orbit difference between PGS-S4 and GEMT1 orbits. 


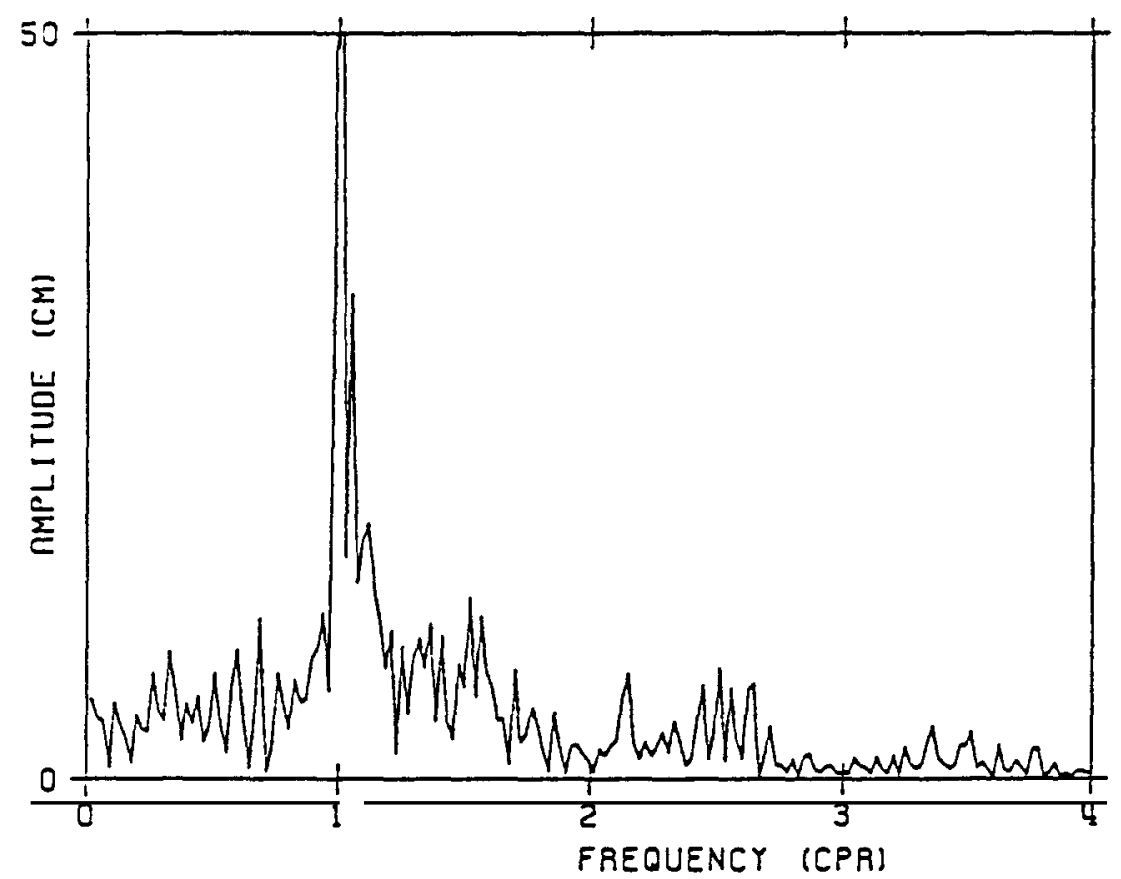

Figure 2. The frequency spectrum of the differences in the radial position component of 3-day SEASAT orbits, computed with the 'PGS-S4 model' and the 'GEM-T1 model' respectively.

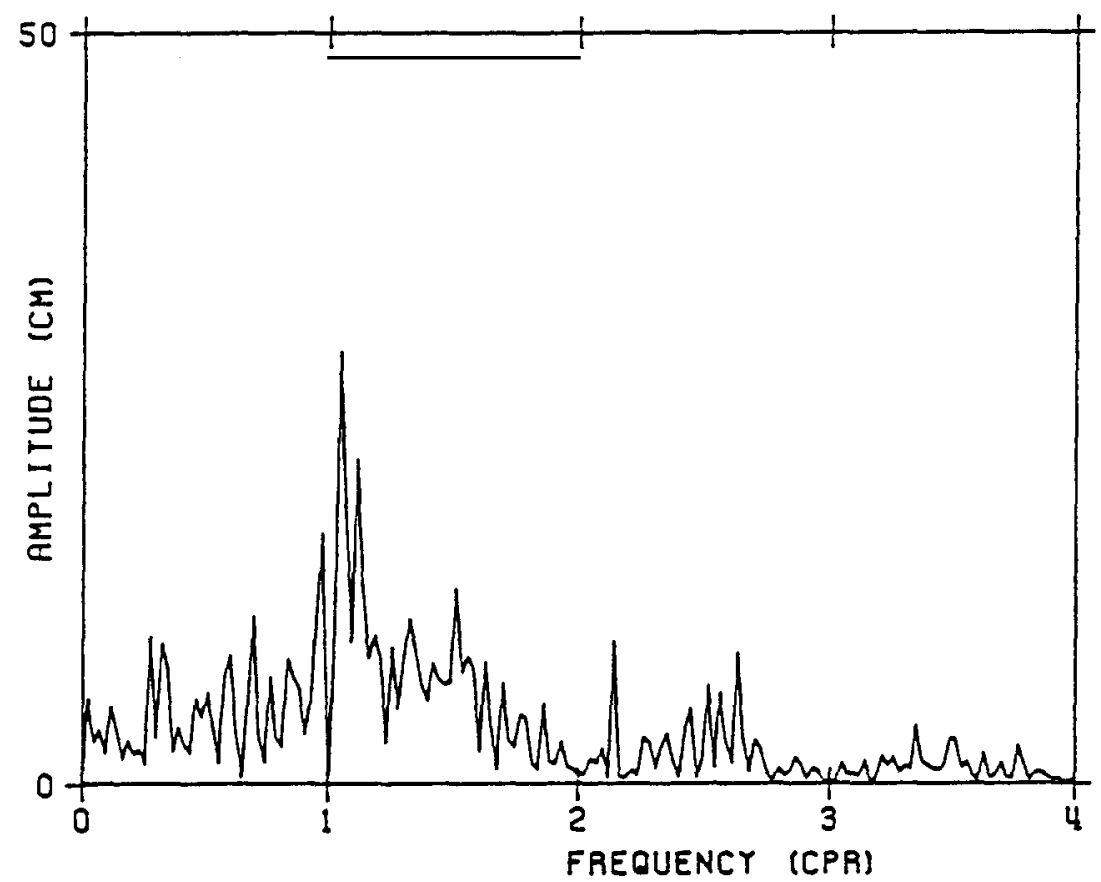

Figure 3. The analytically predicted frequency spectrum of the differences in the radial position component of SEASAT orbits computed with the PGS-S4 and GEM-T1 gravity models. 


\section{GRAVITY FIELD MODEL ERRORS}

There are basically two ways to determine the orbit error due to a gravity model error. The first has been used above, and consists in computing the same satellite orbit with two different models, and comparing the results. This is a very practical approach, but the results must be interpreted with great care. Only if the gravity models have been computed using independent data, the errors of the two models will be uncorrelated. Because most of the recent gravity models depend heavily on the same satellite data, there will always be some correlation. Even if this correlation is low, the difference between the two orbits mainly provides information about the least accurate of the two models, while one would be more interested in the error of the state-of-the-art model.

The alternative approach is to use the covariance matrix obtained during the gravity model computation. This covariance matrix, however, is a formal statistics entity computed from a leastsquares minimization process, and the resulting covariances are based solely upon measurement noise. In other words: the uncertainties in the coefficients given by the covariance matrix are valid under the assumptions that the errors in the data used for the computation are uncorrelated and that the model is able to fully and completely describe the gravity field. In order to use this matrix for a realistic accuracy assessment, it has first to be scaled by comparing it with independent data (surface gravity, resonance constraints etc.). This approach was used in [9] to estimate the accuracy of the GEM-T1 model for TOPEX orbit computation, yielding an $\mathrm{rms}$ value of $25 \mathrm{~cm}$ for the radial component.

Gravity model computation from only satellite tracking data is limited by the fact that the available satellite data do not cover all relevant inclinations and semi-major axes. A gravity model based on these data alone will possess heavily correlated coefficient values, and will be unsuitable for the computation of satellite orbits with other inclinations and semi-major axes. The most common solutions to this uncertainty are the inclusion of additional gravity anomaly information, and collocation, which may be described as a way of constraining the coefficient values. For so-called tailored gravity models, which are meant to be very accurate for one satellite only, there are some additional considerations. Using analytical theory it may be shown that many different terms in the gravity field lead to the same orbit perturbations, and it is therefore possible to select a relatively small subset of gravity model coefficients which may be adjusted relative to an existing gravity model, in order to achieve an improvement in the accuracy of this model for only one satellite. The analytical theory of orbit perturbations due to the gravity field has been presented in many forms $[9,10,11]$. A somewhat alternative appoach has been taken by DUT/SOM, which will be summarized in the following.

\section{ANALYTICAL PREDICTION OF RADIAL ORBIT ERRORS DUE TO THE GRAVITY MODEL}

Analytical computation of satellite orbit errors due to a gravity model error is usually based on Kaula's theory [12], with a number of simplifying assumptions in order to reduce the computational effort. The perturbations of the Keplerian orbital elements resulting from these analyses are then transformed into cross-track, radial and along-track perturbations. In this paper an altemative approach is used. The equations of motion are directly expressed in an in-orbit reference system, and yield a solution with sufficient accuracy for the purposes of this study. This theory also uses a number of simplifying assumptions. For altimeter satellites the most interesting error is that of the radial position component. Because the motion inside the orbital plane and that perpendicular to the orbital plane are only dynamically linked through second-order effects, the equations of motion will be written in a coordinates system in which there are only two components: a radial and a circumferential component. Equations governing the perturbations perpendicular to the plane may be set up and solved separately.

In the derivation it is assumed that the orbital plane has a fixed orientation in space, and a correction is made for this to the Earth's daily rotation velocity. The error thus introduced, caused by the fact 
that the system of integration is no longer inertial, will be negligible as long as the theory is applied to shor test arcs. Now the in-plane equations of motion are described by:

$$
\begin{aligned}
& \ddot{r}-r \dot{\theta}^{2}=-\frac{\partial U}{\partial r} \\
& r \ddot{\theta}+2 \dot{r} \dot{\theta}=-\frac{\partial U}{r \partial \theta}
\end{aligned}
$$

where $r$ is the radius, $\theta$ the in-plane angle and $U$ the total potential which determines the satellite motion. The reference time $(\curvearrowleft=0)$ is chosen at an ascending node passage.

An important approximation made in the following derivation is the assumption that the unperturbed orbit is exactly circular, and that the investigated perturbation is relative to this circular orbit. This means that an error proportional to the orbital eccentricity is made in modeling the perturbing forces. For all past and present altimetry satellites the orbital eccentricity is of the order of 0.001 , so also this approximation will not lead to major errors, again provided that all analyses are performed with relatively short arc lengths (not more than a few days). In the resulting equations $a$ is the semimajor axis of the unperturbed circular orbit and $\omega_{0}$ the mean angular velocity. $\lambda_{a}$ Is the longitude of the ascending node and $\Omega_{c}^{\prime}$ is the earth's daily rotation velocity corrected for the orbital precession. Using Kaula's transform [12] the gravity field may be written as an infinite trigonometric series. Instead of the usual expansion into $G_{\text {to }}(e)$ functions, this study uses the following expression for the potential of one term $U_{b m}$ of the gravity field:

$$
\begin{gathered}
U_{l m}=\frac{\mu}{a}\left(\frac{a_{c}}{a}\right)^{l} J_{l m} \sum_{p=0}^{l} F_{l m p}(I) \cos \left(f_{l m p} \omega_{0} t+m \lambda_{a}-\beta^{*}\right) \\
f_{l m p}=l-2 p-\frac{m}{n^{\prime}} \quad n^{\prime}=\frac{\omega_{0}}{\Omega_{c}^{\prime}}
\end{gathered}
$$

where $J_{m m}$ and $\beta^{*}$ are given by:

$$
\begin{aligned}
& C_{l m}=J_{l m} \cos \beta \\
& S_{l m}=J_{l m} \sin \beta
\end{aligned} \quad \beta^{*}=\left[\begin{array}{cc}
\beta & l-m \text { even } \\
\beta+\frac{\pi}{2} & l-m \text { odd }
\end{array}\right.
$$

Instead of considering the total effect of a term of the gravity field model, one may consider the effect of the error in the values of the coefficients. In the following $\Delta J_{\mathrm{bm}}$ and $\Delta \beta^{*}$ are the values computed from the coefficient enrors $\Delta C_{m}$ and $\Delta S_{l m}$ using Eq.(3). After some straightforward manipulations it appears that:

$$
\Delta_{l m}=a(l-1)\left(\frac{a_{e}}{a}\right)^{l} \Delta_{l m} \sum_{p=0}^{l} \frac{F_{l m p}(I)}{1-f_{l m \varphi}^{2}}\left[1-\frac{2 m}{(l-1) f_{l m p} n^{\prime}}\right] \cos \left(f_{l m p} \omega_{0} t+m \lambda_{a}-\Delta \beta^{*}\right)
$$

With the above theory it is possible to predict the effect of gravity field model errors. If an error model has been defined in some way (for example as the difference between two existing gravity models), it is possible to predict the Fourier series of the resulting radial orbit error using Eq.(4). In that case one is only interested in the amplitudes for the given frequencies $\left(f_{l m p} \omega_{0}\right)$, without looking at the phase of the error signal contributions. An example of this application is given in Figure 3, which shows the predicted radial orbit error spectrum for SEASAT, using a simulated gravity model error equal to the difference between the PGS-S4 and GEM-T1 gravity models. Comparing this with Figure 2, a good correspondence is found, except for the term with exactly one cycle per revolution, which cannot be estimated using this theory. It should also be noted that Figure 2 did not pertain to GEM-T1 but a slightly different preliminary version. 


\section{ESTIMATION OF GRAVITY COEFFICIENTS USING CROSS-OVER DATA AND CONSTRAINTS.}

For SEASAT orbit computation the general-purpose model GEM-T1 is already an improvement over the tailored model PGS-S4, but still the radial accuracy is insufficient for a direct application of the SEASAT altimeter data to ocean dynamics studies. This is even more true for ERS-1, as Rosborough [9] indicates that the expected radial orbit accuracy of GEM-T1 for this satellite is noticeably inferior. It has therefore been investigated by DUT/SOM what accuracy improvement of GEM-T1 could be achieved by tailoring it to ERS-1, while SEASAT was used as a stand-in for ERS-1. As previous experiments have shown $[7,8]$, a subset of 60 coeficients is insufficient for a significant improvement, but at the same time the laser tracking data of four 3-day SEASAT arcs are insufficient for the determination of a larger subset. Altimeter tracking data should not be used globally for this purpose, as the sea surface topography may be aliased into the recovered gravity model. For this reason cross-over data were selected to serve as tracking data in addition to the available laser range observations, while the singularity of the normal equations was reduced by setting up a constraint matrix which was to be added to the normal equations.

From Figures 2 and 3 it can be seen that orbit perturbations due to gravity model errors with frequencies above two cycles per revolution may be kept out of consideration because their effect is much smaller than that of lower frequencies. This is due to the attenuation factor $\left(1-f_{\operatorname{lmp}}{ }^{2}\right)$ seen in the denominator in Eq.(4), which also appears in the relations for the cross-track and along-track perturbations. The equations also show that the frequencies occurring in the radial and along-track perturbations only depend on the gravity term order $m$ and the parity of the degree $l$.

Because laser range observations provide a combination of cross-track, radial and along-track information, it will not suffice to allow only improvement in the radial direction. A minimization of laser residuals is only possible if the orbit may be improved in all three components. Eq.(2) shows that every pair of coefficients produces two frequencies between zero and two cycles per revolution in the radial and along-track perturbations. For the cross-track perturbations the frequencies are given by:

$$
f_{l m r}=l-2 r-1-\frac{m}{n^{\prime}}
$$

These are the same frequencies that occur in the radial and along-track directions (except that they occur at the opposite parity of the degree, which has no influence on the selection procedure used here). The conclusion is that in order to cover all frequencies between zero and two cycles per revolution in all three direction components, a total of twelve pairs of coefficients have to be estimated for each order of the gravity field model, six for odd degrees and six for even degrees.

\section{ADUSSTMENT OF GEM-TI COEFFICIENTS FOR ORBIT ERROR IMPROVEMENT}

In order to limit the number of coefficients to be estimated it was decided to truncate the number of orders for which coefficients were to be adjusted. Figure 4, which was taken from Rosborough [9], shows the expected radial error of TOPEX orbits computed with GEM-T1 as a function of the coefficient order. This figure was deduced from the covariance matrix of the GEM-TI solution. It shows that above order 13 the remaining error is limited. Although Figure 4 is not directly applicable to SEASAT, it is assumed that the major errors for this satellite will be due to the coefficients with order less than or equal 15 , being one more than the number of revolutions per day $\left(n^{\prime}\right)$ for this satellite. With 12 coefficient pairs per order, this leads to a total of 360 coefficients to solve. It was also decided not to adjust any zonal harmonics. The selected set of coefficients is shown schematically in Figure 5. 


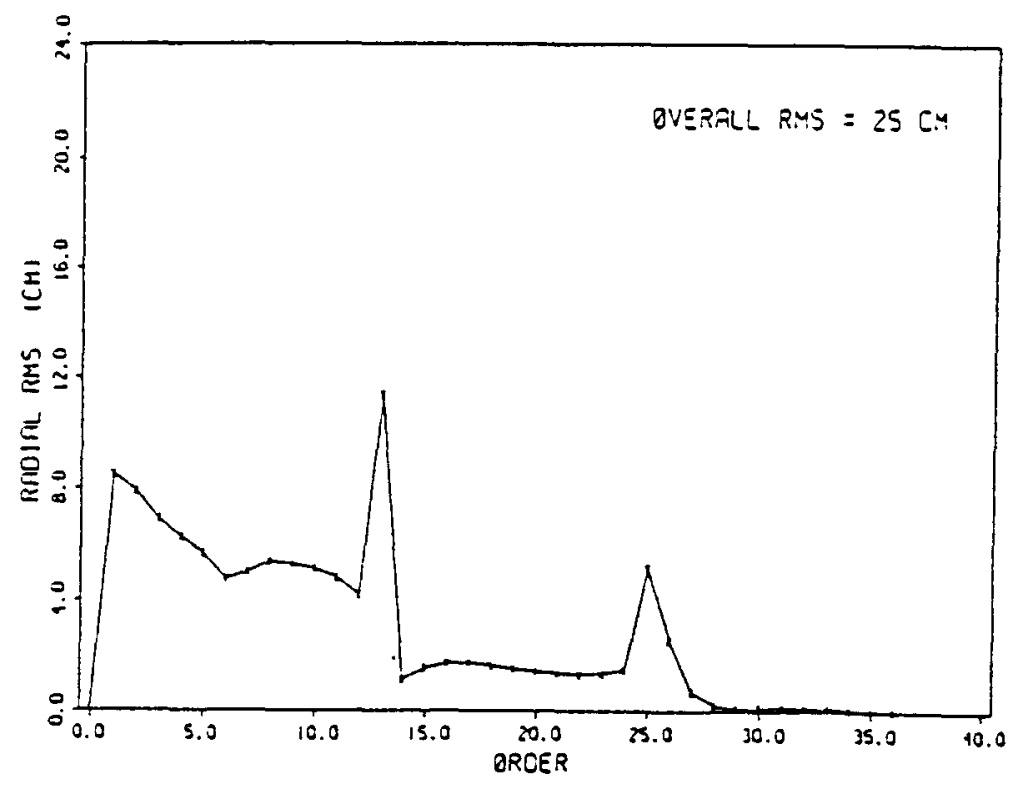

Figure 4. Rms of the TOPEX radial orbit standard deviation as a function of gravity term order $m$, for the GEM-T1 gravity model, deduced from the variance-covariance matrix (from Rosborough [9]).

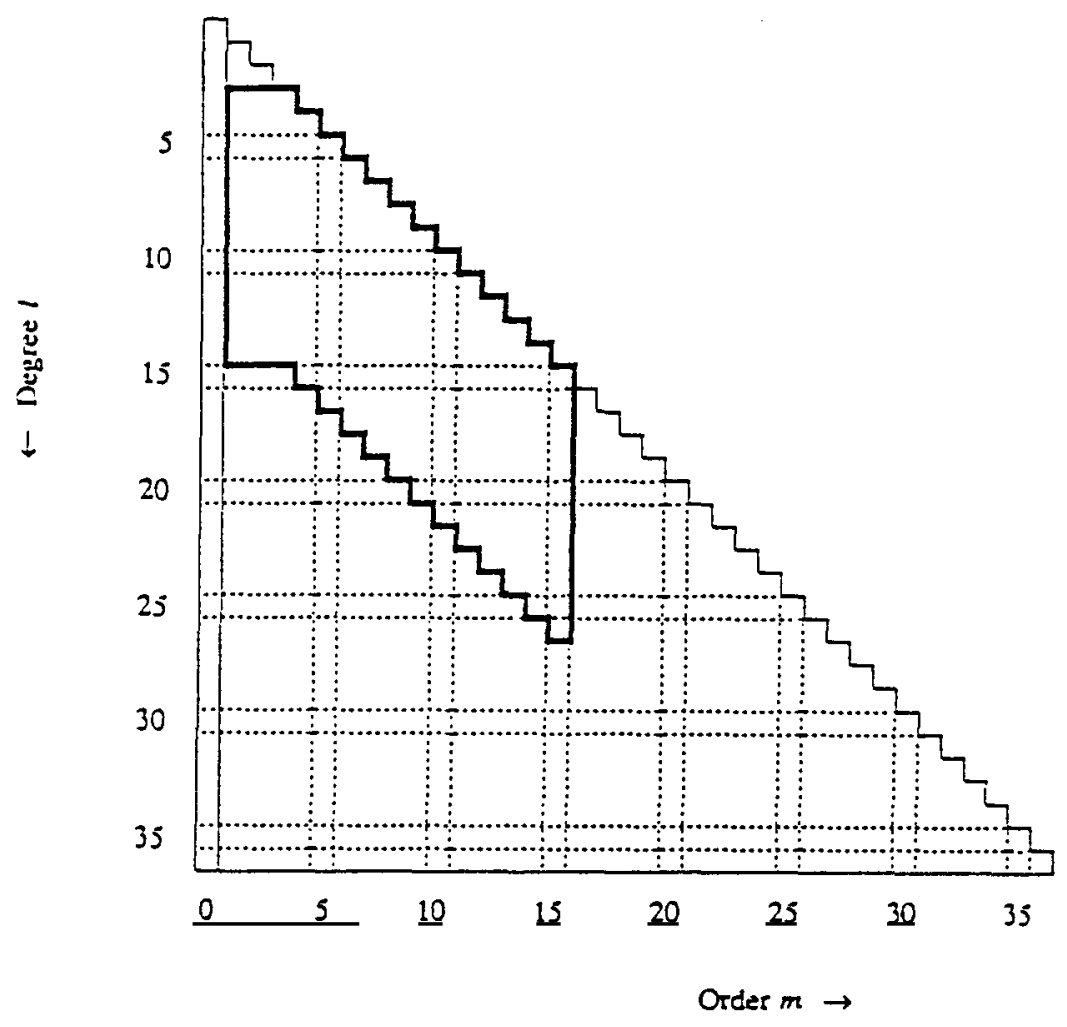

Figure 5. Gravity coefficients selected for the gravity model tuning experiment. This figure represents the complete $36 * 36$ GEM-T1 model, while the selected coefficients have been surrounded by a heavy border. 
Four SEASAT data ares of 3 days each were available for the adjustment process. Some information about these arcs is listed in Table 3, together with additional information about the adjustment process. The arc lengths and number of laser and cross-over observations being insufficient for a straightforward solution, it was decided to add constraints to the system of equations, by requiring that wherever tracking data are insufficient, the orbit should remain as close as possible to the original GEM-TI orbit, which represents the best current solution. To achieve this, state vector data were generated on the original GEM-Tl orbits, and normal equations were set up for fitting the orbit to these observations. Normal equations were also set up for the laser and cross-over data of the four arcs, and the coefficients were solved by combining the matrices with various weight ratios, using two iterations.

It appeared that the weight ratio had a large impact on the resulting coefficient adjustments. Of course, if the constraint weight is infinite, all adjustments will be zero, and if the weight is zero the adjustments will be unrealistically large. The weight was adjusted empirically, checking the coefficient adjustments with Kaula's rule of thumb. This is illustrated by Figure 6 . This figure shows the ms values of the coefficient adjustments for each gravity term order $m$, divided by the value according to Kaula's rule. The weight ratio for which the rms adjustment values were just below 1 was chosen for the final adjustment. In the following the adjusted GEM-T1 model after 2 iterations will be designated by GEM-TICX, where the $X$ symbolizes the use of cross-over observations during the computation of the model. The second iteration was performed without updating the constraint matrix, because the orbit adjustment was only minor.

Table 3. Summary of the models and the SEASAT tracking data applied in the gravity model tuning experiment.

\begin{tabular}{|c|c|}
\hline GRAVITY & Base model: GEMI-T1 \\
\hline MODEL & $\begin{array}{l}\text { - } 180 \text { pairs of C,S coefficients estimated } \\
\text { - } \quad \text { "Infinite" a priori standard deviations } \\
\text { - Constraints based on GEM-TI a priori orbit }\end{array}$ \\
\hline CONSTANTS & $\begin{array}{l}\text { - } a_{e}=6378.137 \mathrm{~km} ; 1 / \mathrm{f}=298.257 ; G M=398600.436 \mathrm{~km}^{3} / \mathrm{s}^{2} \\
\mathrm{c}=299792.458 \mathrm{~km} / \mathrm{s}\end{array}$ \\
\hline $\begin{array}{l}\text { TRACKING } \\
\text { DATA }\end{array}$ & $\begin{array}{l}\text { - Four nearly consecutive } 3 \text {-day data arcs in the 3-day } \\
\text { repeat orbit, containing } 96 \text { laser ranging passes over } \\
10 \text { stations. Normal points weight: } 1 \mathrm{~m} \text {. } \\
\text { - SL6 station coordinates scaled to adopted value of ae. } \\
\text { - } 2831 \text { Cross-over differences, weight: } 1 \mathrm{~m} \text {. } \\
\text { - One 3-day test arc in the } 17 \text {-day repeat orbit. } \\
\text { - One 3-day test arc in the 3-day repeat orbit, high } \\
\text { solar activity. }\end{array}$ \\
\hline $\begin{array}{l}\text { OTHER } \\
\text { MODELING }\end{array}$ & $\begin{array}{l}\text { - MSIS (1983) atmospheric drag, daily drag coefficients } \\
\text { adjusted. }\end{array}$ \\
\hline INFORMATION & $\begin{array}{l}\text { - Solar radiation pressure, arc-dependent pressure } \\
\text { coefficient adjusted. } \\
\text { Luni-solar attraction, solid earth tides, precession, } \\
\text { nutation, polar motion, UT1 and indirect moon-J2 } \\
\text { effects all applied. }\end{array}$ \\
\hline INTEGRATION & - 11-th order Cowell integration, stepsize $75 \mathrm{~s}$. \\
\hline
\end{tabular}


Table 4. Laser and cross-over difference residuals for four 3-day SEASAT arcs computed with the GEM-T1 model and with the adjusted GEM-TICX model. Values are mean/rms in $\mathrm{cm}$. In all cases both laser and cross-over data were used together as tracking data.

\begin{tabular}{ccccc}
\hline Arc & \multicolumn{2}{c}{ Laser residuals } & \multicolumn{2}{c}{ Cross-over residuals } \\
ID & GEM-T1 & GEM-T1CX & GEM-T1 & GEM-T1CX \\
\hline C2 & $-7 / 65$ & $0 / 45$ & $4 / 63$ & $2 / 42$ \\
C3 & $9 / 47$ & $3 / 28$ & $7 / 62$ & $5 / 40$ \\
C4 & $-8 / 49$ & $-3 / 30$ & $7 / 60$ & $4 / 37$ \\
C5 & $-12 / 62$ & $-6 / 36$ & $4 / 62$ & $2 / 38$ \\
\hline
\end{tabular}

Table 5. Laser and cross-over difference residuals for two 3-day SEASAT test arcs computed with the GEM-T1 and GEM-T1CX models. Test arcs $\mathrm{Cl}$ and $\mathrm{C} 6$ lie in the 17-day and 3-day repeat periods respectively. Values are $\mathrm{rms}$ in $\mathrm{cm}$. In all cases both laser and cross-over data were used together as tracking data.

\begin{tabular}{lcccc}
\hline Arc & \multicolumn{2}{c}{ Laser residuals } & \multicolumn{2}{c}{ Cross-over residuals } \\
ID & GEM-T1 & GEM-T1CX & GEM-T1 & GEM-T1CX \\
\hline C1 & 54 & 52 & 68 & 57 \\
C6 & 121 & 118 & 93 & 66 \\
\hline
\end{tabular}

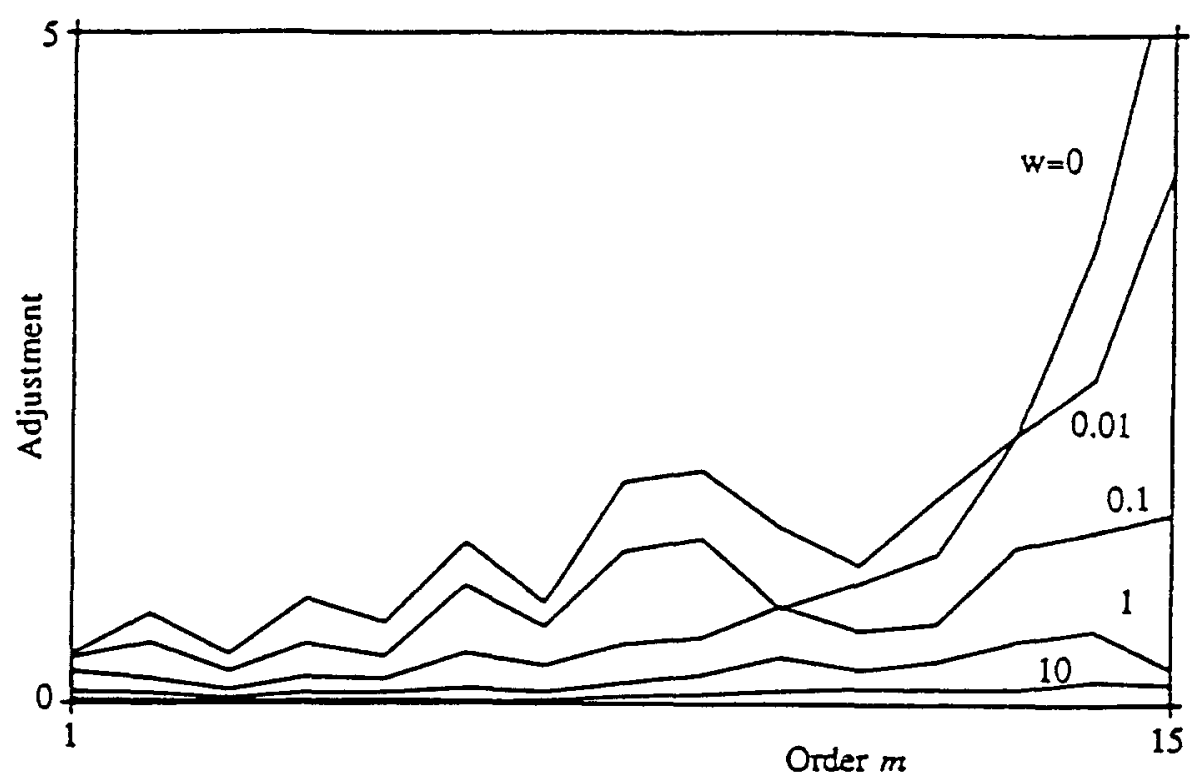

Figure 6. The influence of the weight of the constraint matrix on the rms adjustment per gravity term order $m$ of the selected coefficients. Each line represents a different relative weight. The values have been divided by Kaula's rule of thumb. 
Table 4 shows the laser and cross-over residual statistics for both the original GEM-T1 model and GEM-T1CX, for the four arcs used in the computation of the new model, using both laser and crossover observations as tracking data. These arcs, designated by $\mathrm{C} 2$ to $\mathrm{C5}$, all lie in the period that SEASAT was in its three-day repeat orbit. It appears that a significant improvement over the GEM. T1 statistics has been achieved, where the improvement during the second iteration was marginal though consistent. The final cross-over residual statistics of 37 to $42 \mathrm{~cm}$ indicates that the remaining radial orbit errors are probably around $30 \mathrm{~cm} \mathrm{~ms}$, and this seems a reasonable value, taking into account the various restrictions in the experiment. The laser residual ms values of 28 to $45 \mathrm{~cm}$ indicate that the total position component has a comparable error, at least during the periods that the satellite was tracked. Because of the limited quality of the data of some of the tracking stations in 1978, it is questionabe whether these figures may yet be improved by much.

Table 5 shows the laser and cross-over residual statistics for two test arcs that were not used during the computation of GEM-TICX. Of these arcs the one designated by $\mathrm{Cl}$ lies in the 17-day nearrepeat orbit, while arc $\mathrm{C} 6$ immediately follows arc $\mathrm{C} 5$, but falls within a period of high solar activity. The relatively minor improvement for arc $\mathrm{Cl}$ of both laser and cross-over residuals indicates that the GEM-T1CX model is also valid for the period that SEASAT was in its 17-day repeat period. There is a noticeable improvement in arc $\mathrm{C} 6$, but the drag modeling problem is probably responsible for the still relatively large residual $\mathrm{ms}$ values.

The main question emanating from this experiment is of course whether the same results may be obtained for ERS-1 when that satellite is in orbit. Because at no point in the experiment explicit SEASAT parameters were used, it has to be assumed that the same accuracy may be obtained for ERS-1 orbits. The only difference is that GEM-T1 is already a very accurate model for SEASAT orbit computation, and this will not be the case for ERS-1. Therefore the constraining procedure used in this experiment may not be applicable then. But at that time the state-of-the-art gravity model GEM-Tn will be very accurate for TOPEX/Poseidon orbit computation, and the constraint matrix may be set up for this satellite. Especially if also ERS/TOPEX cross-over differences will be used as tracking data, it must be avoided that the GEM-Tn model deteriorate for TOPEX/Poseidon as a result of the tailoring process.

\section{A POSTERIORI ORBIT ERROR CORRECTION}

Whereas the precision of the radar altimeter height observations after applying the necessary media corrections is well below $10 \mathrm{~cm}$ for current and future radar instruments, the error introduced by the orbit computation reduces the absolute accuracy of the resulting sea height above the reference ellipsoid to some $50 \mathrm{~cm}$ for SEASAT orbits, when state-of-the-art geophysical models are applied. As a result of this, the radial orbit error has to be eliminated in some other way. Some very useful techniques to achieve this have been devised in the past decade, using which accurate oceanographic results have been obtained [13,14]. One of these techniques is commonly called the local cross-over minimization technique, and consists in the removal of a linear trend from altimeter residuals on short arcs (up to $2000 \mathrm{~km}$ ) in a restricted area, in such a way that that the cross-over residuals are minimized in a least-squares sense $[15,16,17]$. This technique requires the subdivision of the global oceans into small patches, to each of which the procedure may be applied, after which the patches may be combined into a global map of the mean sea surface. As a result of this, the basin-wide topography is lost. If, by using a more realistic orbit error model, the maximum size of the area could be increased, the loss of basin-wide information would be reduced. The following section will introduce a simple though effective improvement in the orbit error correction function, and discuss how it has been implemented in the DUT/SOM software system. 


\section{AN IMPROVED ORBIT ERROR CORRECTION PROCEDURE}

As shown above (Figures 2,3) a Fourier analysis of a typical radial orbit error has the highest amplitudes near the frequency of one cycle per orbital revolution. Because of this fact, during part of an orbital revolution the error may very well be approximated by a sinusoidal function with a frequency of one cycle per revolution.

If we write:

$$
\rho(t)=c(t) \cos u+s(t) \sin u
$$

where $p(t)$ represents the actual (and unknown) radial orbit error as a function of time, and $u$ is the argument of latitude, or the in-orbit angle between the satellite position and the most recent pass through an ascending node, then, for a typical radial orbit error signal, boch $c(t)$ and $s(t)$ will be slowly-varying functions of time. As a consequence these functions will be almost constant during periods shorter than one orbital revolution [18].

A useful application of this is found in the local cross-over minimization procedure. Instead of representing an orbit error on a short arc by two parameters defining a linear function, it may be represented by two constant values $\bar{c}$ and $\bar{s}$, resulting in the efror function:

$$
\bar{\rho}=\bar{c} \cos u+\bar{s} \sin u
$$

This enror function may be used over longer arcs than the conventional linear function, as will be demonstrated in the following section.

It should be noted here that the separation of the radial orbit error signal into two functions, as given in Eq.(6), would seem to lead to a fundamental problem: for any given orbit error signal there is an infinite number of possible separations into functions $c(t)$ and $s(t)$. This does not mean that the error correction in Eq.(7) cannot be determined. In this relation the parameters $\bar{c}$ and $\bar{s}$ are imposed to be constant, and they refer to the one separation of Eq.(6) which is smoothest according to a certain smoothness criterium. In other words: among the infinite number of possible separations of $p(t)$ into $c(t)$ and $s(t)$, one combination may be found for which $c(t)$ and $s(t)$ are most nearly constant during the short pass under consideration.

An interesting aspect of the use of sinusoidal orbit error corrections is that the computed values of $\bar{c}$ and $\bar{s}$ for each of the tracks should form a long-wavelength time series. Furthermore, if the eliminated radial orbit error for a certain test area is only due to the gravity model error, the values of $\bar{c}$ and $\bar{s}$ recovered from different test areas should lie on the same curves. This leads to the following interesting points:

- Graphs of the values of $\bar{c}$ and $\bar{s}$ as a function of time should be similar for all test areas. By comparing these graphs, outliers due to other error sources may be identified.

- By solving the values of $\bar{c}$ and $\bar{s}$ for several areas, reliable functions $c(t)$ and $s(t)$ may be computed by interpolation, which may be interpreted as the actual (uncorrelated) radial orbit error due to the gravity model error. The application of this function is two-fold:

- Imposing these functions as the track corrections instead of the previously computed $\bar{c}$ and $\bar{s}$, will automatically lead to smooth transitions between adjacent test areas.

- The radial orbit error $p(t)$, restored from $c(t)$ and $s(t)$, yields significant information about the remaining gravity model error.

This new cross-over minimization procedure has recently been implemented at DUT/SOM, and the first results will be discussed in the following. 


\section{APPLICATION TO AN IMPROVED CROSS-OVER MINIMIZATION PROCEDURE}

The sinusoidal cross-over minimization procedure has been set up modularly so that optimal use can be made of the above-mentioned advantages of the error correction model. This is demonstrated in Figure 7 which shows the procedure in a simplified block diagram. Indicated are the possibilities of feeding back the computed error correction, and combining these for several regions. The procedure is also capable of treating data from various satellites supporting different specifications of radial orbit accuracy and altimeter noise level. The cross-over minimization procedure has been applied to a test area in the Southern Atlantic Ocean, from $30^{\circ} \mathrm{W}$ to $10^{\circ} \mathrm{E}$ and from $5^{\circ}$ to $45^{\circ} \mathrm{S}$. This $40^{\circ} *$ $40^{\circ}$ area, with an approximate size of $3500 * 4000 \mathrm{~km}^{2}$, comprises such topographic features as the Mid-Atlantic Ridge and the Walvis Ridge near Africa. This area has also been subdivided into four equal sections of $20^{\circ} * 20^{\circ}$ (Figure 8 ), and the new minimization procedure has been applied to both the total area and the four sub-areas.

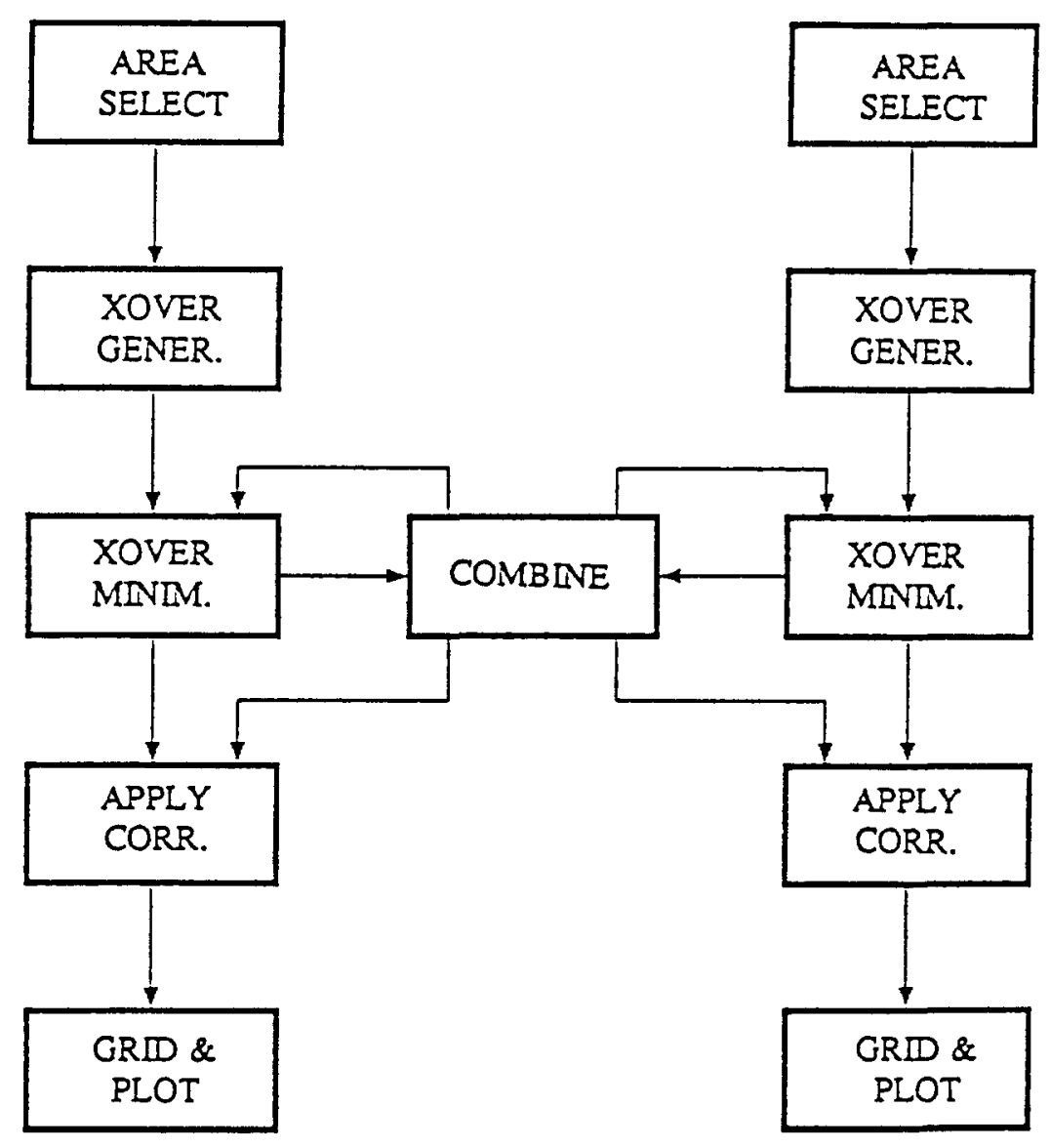

Figure 7. The improved local cross-over minimization procedure. The two piles represent identical applications of the procedure to different areas: selection of altimeter data for an area, generation of cross-overs, minimization of cross-over residuals, correction of altimeter data, gridding and plotting. The central module combines and adjusts the track correction parameters. The adjusted values may be used as a priori values for a new minimization step, or applied instead of the previous values. 

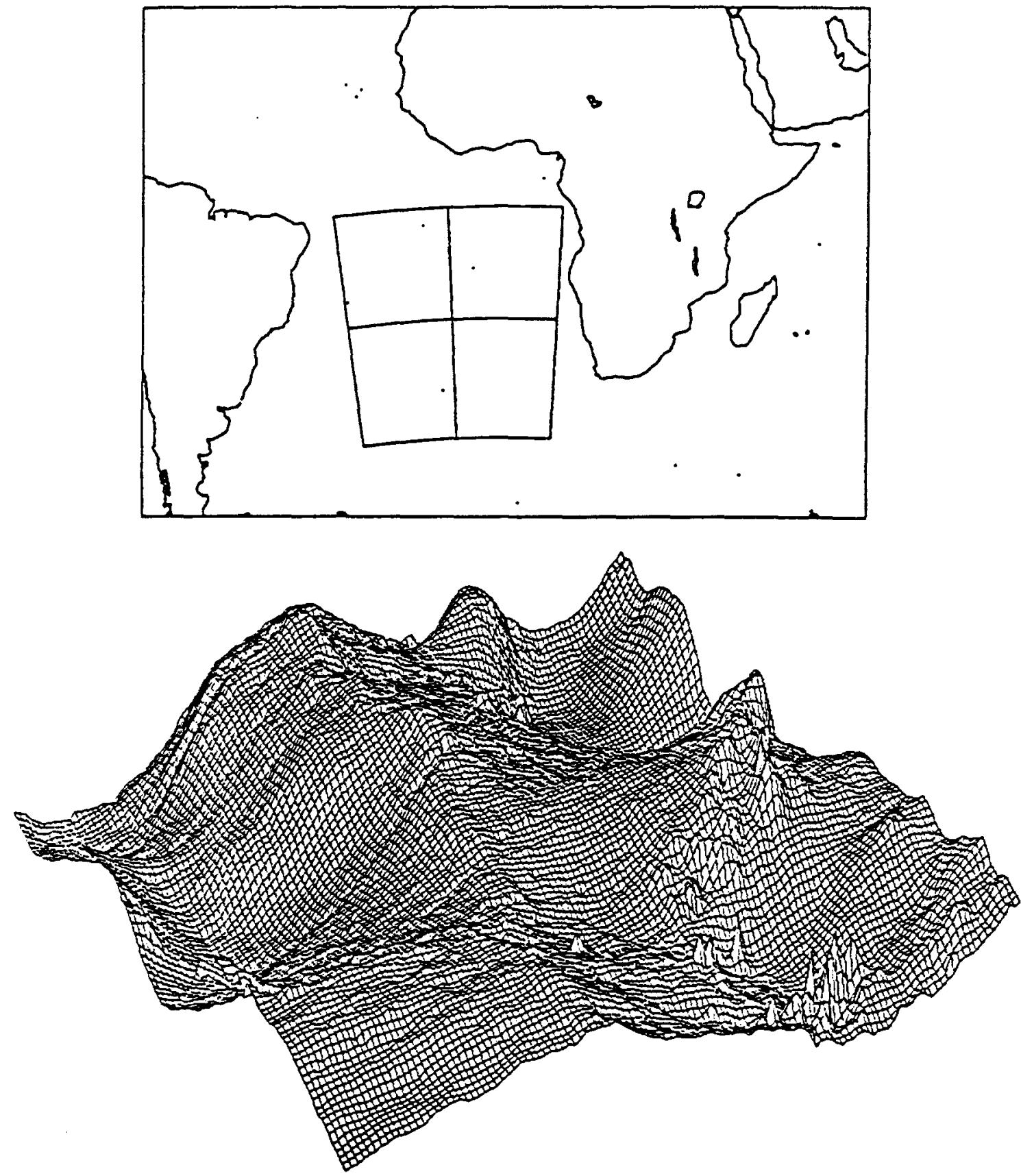

Figure 8. Application of the local cross-over minimization procedure to a test area in the South Atlantic (longitude $30^{\circ} \mathrm{W}$ to $10^{\circ} \mathrm{E}$, latitude $5^{\circ}$ to $45^{\circ} \mathrm{S}$ ), which has also been subdivided into four $20^{\circ} * 20^{\circ}$ blocks (top figure). The bottom figure shows a 3-dimensional representation of the recovered mean sea surface relative to a $12 * 12$ truncation of the GEM-10B geoid model, viewed from the south-west. Clearly visible are the Mid-Atlantic ridge (center) and the Walvis ridge (right). 
It is a well-known phenomenon that a least-squares cross-over minimization procedure does not have a unique solution. In fact, if two parameters per track, defining a long-wavelength correction function, are used, four degrees of freedom may be identified, resulting in four Eigenvalues of 0 or almost 0 in the system of normal equations. These must be overcome in order to find a solution, preferably the most likely solution. As part of the experiments two methods of overcoming the degrees of freedom have been tested. The first of these is a Bayesian approach, where each of the coefficients $\bar{c}$ and $\bar{s}$ has an a priori value of zero and a non-zero standard deviation. The second is a much less stringent functional approach where the degrees of freedom are expressed as four Legendre functions of latitude and longitude, which are minimized in a least-squares sense over the test region. It appeared that the latter method still left relatively ill-conditioned normal equations. The results of the new procedure were also compared with the traditional linear orbit error correction method.

The cross-over residual statistics of these cases are gathered in Table 6. This table shows that the sinusoidal procedure with Bayesian constraints leaves a cross-over ms for the total area which is of the same magitude as the sub-areas, while the traditional linear method breaks down for the larger area, even though the results for the sub-areas are of the same quality as with the sinusoidal method. Another interesting point is the low value of the cross-over residual ms for the functional constraint. This shows that the oceanic variability is relatively low in this area, and that the a posteriori statistics for the other solution methods still contain a residual orbit error. Although the functional constraint leaves the lowest cross-over residuals, the resulting mean sea surface topography is not the most reliable. Investigation of the absolute position of the sub-areas relative to the total area has indicated that the solution is unstable. For the Bayesian constraint there was a better correspondence, and therefore a more reliable solution. This solution is shown in a 3-dimenisional view in Figure 8, which clearly displays the ocean topography due to the Mid-Atlantic Ridge and the Walvis Ridge in very fine detail.

Table 6. Cross-over residual ms after applying a regional least-squares cross-over minimization procedure. The total area has a size of $40^{\circ} * 40^{\circ}$, and has been subdivided into four quarters of $20^{\circ} *$ $20^{\circ}$ each. Two constraining methods were used for the sinusoidal correction model: a Bayesian constraint of $1 \mathrm{~m}$ and a very weak functional constraint. The results are compared with the traditional linear method.

\begin{tabular}{lcccc}
\hline Area & $\begin{array}{c}\text { Size } \\
\text { (deg*deg) }\end{array}$ & \multicolumn{3}{c}{ A posteriori cross-over rms (cm) } \\
& $\begin{array}{c}\text { Sinusoidal } \\
\text { Bayesian }\end{array}$ & $\begin{array}{c}\text { Sinusoidal } \\
\text { Functional }\end{array}$ & $\begin{array}{c}\text { Linear } \\
\text { (Bayesian) }\end{array}$ \\
\hline Total & $40^{*} 40$ & 11 & 9 & 15 \\
& & & & \\
Sub 1 & $20^{*} 20$ & 8 & 5 & 8 \\
Sub 2 & $20^{*} 20$ & 8 & 4 & 8 \\
Sub 3 & $20^{*} 20$ & 11 & 7 & 13 \\
Sub 4 & $20^{*} 20$ & 12 & 9 & 11 \\
\hline
\end{tabular}


It was also tested whether the $\bar{c}$ and $\bar{s}$ coefficients recovered from two different oceanic regions after a cross-over minimization could be compared. This is shown in Figure 9, where the recovered values are plotted as a function of time for an arbitrarily selected time interval. The test regions lie in the Atlantic and Pacific Oceans respectively. It appears that there is both a common signal and a noiselike constituent. With software that is currently being developed by DUT/SOM, a common radial orbit ertor signal may be deduced from these data, which then may be fed back into the Bayesian minimization procedure as a priori values, to obtain a second iteration. The common signal in Figure 9 shows a period of approximately 14 days, which indicates a 14-day modulation of the 1 cycle-per-revolution radial orbit error. This would seem to indicate that a significant part of the recovered radial orbit error may be due to tides, but because the arc length used in the orbit computation in this period was 6 to 7 days, it is difficult to judge whether the effect is real or artificial.
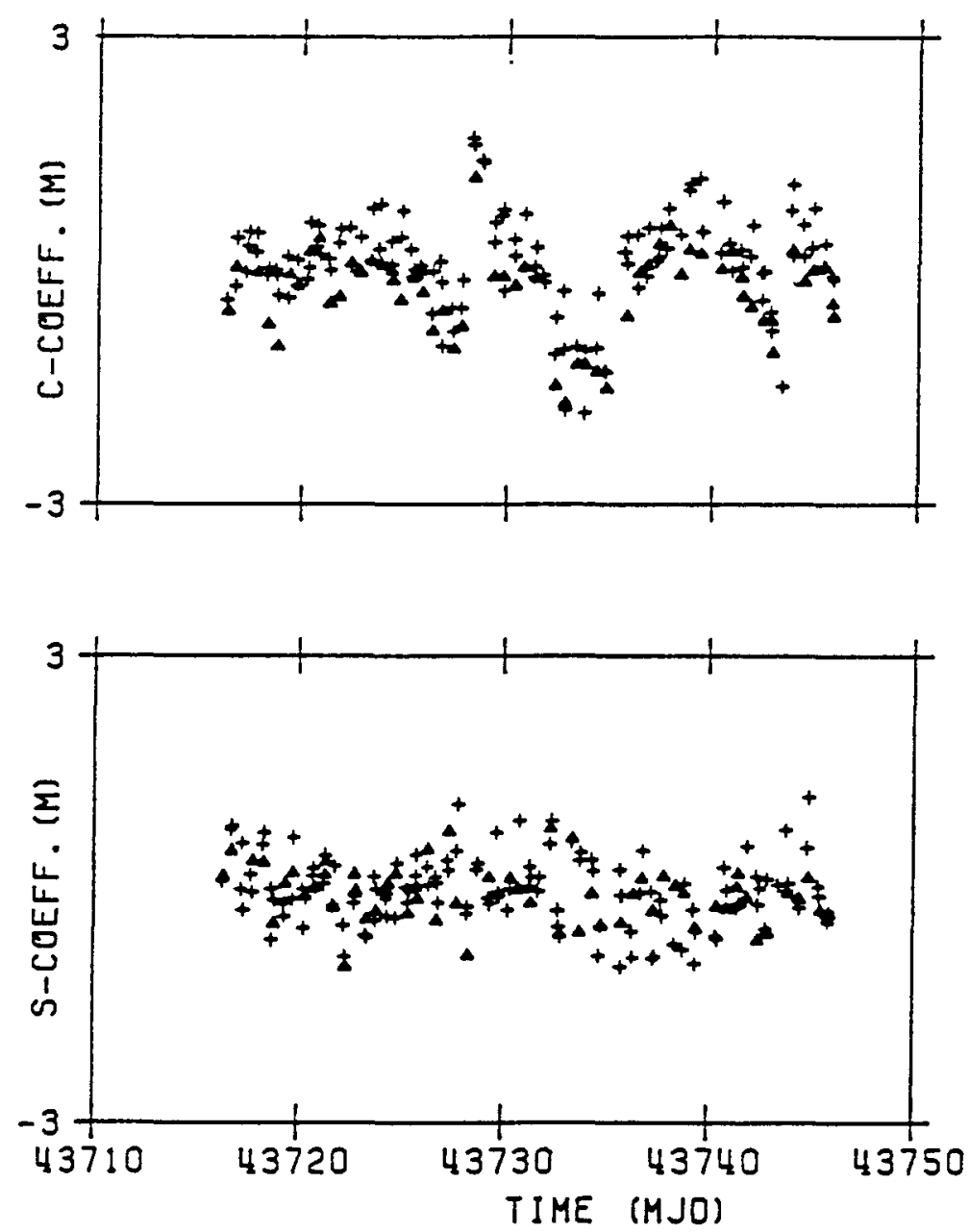

Figure 9. The recovered track correction parameters $\bar{c}$ and $\bar{s}$ (in $\mathrm{m}$ ) of a local cross-over minimization procedure, applied to two different test areas, indicated by triangles and crosses. Although the areas lie in different oceans, a common signal may be seen in both sequences. The low-frequency contribution to the $\bar{s}$-coefficients may be an indication of a tides modeling error. 


\section{FNAL CONCLUSIONS}

In the study described in this paper a SEASAT-tailored version of the GEM-T1 gravity model has been created based on four 3-day data arcs, and laser and cross-over tracking data. This tailored model called GEM-TICX does not pretend to be a very good model in the sense that it accurately describes the gravity field of the Earth, but it does produce SEASAT orbits which coincide very well with the available tracking observations. The ultimate goal of this study is to set up a processing scheme which is able to produce an ERS-1-tailored gravity model after only a short period of time, and to be able to update the model as soon as more data become available. In order to enable the combination of altimetry data from ERS-1 and TOPEX/Poseidon, this tailored model should not just be tailored to ERS-1, but should retain the quality of the then available general purpose model GEM-T $n$ when used for TOPEX/Poseidon orbit computation.

The results, yielding an estimated global radial orbit error of $30 \mathrm{~cm}$ for SEASAT, are encouraging, but it has to be remembered that in the early 1990's tracking data, station positions and atmospheric models will be more accurate, so that a further improvement should be possible. If the ultimate goal remains the $10 \mathrm{~cm}$ for the radial component, additional gravity coefficients will have to be adjusted, the constraint for the original ERS-1 orbit has to be lessened and TOPEX orbit constraints should be added. With these modifications the effort comes more and more close to that of the adjustment of an entire gravity model. The next objective is therefore to find out what is the limit of orbit improvement by adjusting only a subset of the gravity model coefficients.

With the sinusoidal cross-over minimization procedure DUT/SOM now possesses an important tool for altimeter data processing. DUT/SOM is not of the opinion that the sinusoidal correction by itself is better than a quadratic error model, which is the most commonly used alternative to the traditional tilt/bias method. The main advantage is the fact that the recovered parameters allow an easy combination of areas and the reconstruction of the global radial orbit error. This is combined with general advantages, not due to the improved error model, but to additional flexibility by the modular setup, the possibility to combine data from satellites with different accuracies and the possibility of backsubstitution of adjusted parameters.

The sea surface variability results obtained in the South Adlantic indicate that the technique applied to overcome the degrees of freedom inherent to the cross-over minimization process, has a strong impact on the resulting variability maps, and that any constraints on track parameters should be as week as possible.

\section{REFERENCES}

[1] F.J. Lerch, J.G. Marsh, S.M. Klosko and R.G. Williamson. Gravity model improvement for SEASAT, J. Geophys. Res., Vol. 87, No. C5, April 1982, pp. 3281-3296.

[2] L.G. Agrotis, J.M. Dow and G. Lecohier. Orbit determination for altimetry missions, Adv. Space Res., Vol. 6, No. 9, 1986, pp. 177-182.

[3] K.F. Wakker, R.C.A. Zandbergen and B.A.C. Ambrosius. SEASAT orbit determination experiments in preparation for the ERS-1 altimetry mission, AAS/ALAA Astrodynamics Specialist Conference, Paper 87-426, Kalispell, August 1987.

[4] J.G. Marsh. GEM-10B $1^{\circ}$ by $1^{\circ}$ global detailed gravimetric geoid, Private communication, November 1985.

[5] C.K. Tai and C. Wunsch. Absolute measurements by satellite altimetry of dynamic topography of the Pacific Ocean, Nature, Vol 301, Februari 1983.

[6] J.G. Marsh, F.J. Lerch, B.H.Putney, D.C. Christodoulidis, T.L. Felsentreger, B.V. Sanchez, D.E. Smith, S.M. Klosko, T.V. Martin, E.C. Pavlis, J.W. Robbins, R.G. Williamson, OL. Colombo, NL. Chandler, K.E. Rachlin, G.B. Patel, S. Bhati and D.S. Chinn. An improved model of the earth's gravitational field: GEM-T1, NASA Technical Memorandum 4019, Goddard Space Flight Center, Greenbelt, 1987.

[7] R.C.A. Zandbergen, K.F. Wakker and B.A.C. Ambrosius. Analysis of radial orbit errors of 
ERS-1 and the development of super-tailored gravity models, Adv. Space Res., Vol. 6, No. 9 , 1986, pp. 183-194.

[8] K.F. Wakker, B.A.C. Ambrosius, R.C.A. Zandbergen and G.H.M. van Geldorp. Precise orbit computation, gravity model adjustment and altimeter data processing for the ERS-1 altimetry mission, ESOC contract study, Delft Univ. Techn., Faculty Aerospace Engin., February 1987.

[9] G.W. Rosborough, Orbit error due to gravity model error. AAS/AIAA Astrodynamics Specialist Conference, paper 87-534, Kalispell, August 1987.

[10] C.A. Wagner. Radial variations of a satellite orbit due to gravitational errors: Implications for satellite altimetry, J. Geophys. Res., Vol. 90, No. B4, March 1985, pp. 3027-3036.

[11] B.D. Tapley and G.W. Rosborough. Geographically correlated orbit error and its effect on satellite altimetry missions, J. Geophys. Res., Vol. 90, No. C6, November 1985, pp. 11817. 11831.

[12] W.M. Kaula. Theory of Satellite Geodesy, Blaisdell Press, Waltham Massachusetts, 1966.

[13] D.T. Sandwell, D.G. Milbert and B.C. Douglas. Global nondynamic orbit improvement for altimetric satellites, J. Geophys. Res., Vol. 91 No. B9, August 1986, pp 9447-9451.

[14] C.K. Tai and L.L. Fu. On cross-over adjustment in satellite altimetry and its Oceanographic Implications, J. Geophys. Res., Vol. 91 No. C2, Februari 1986, pp 2549-2554.

[15] J.G. Marsh, R.E. Cheney, T.V.Martin and JJ. McCarthy. Mean sea surface computations in the northwest Pacific based upon satellite altimeter data, Proc. General IAG Meeting. Tokyo, May 1982, pp. 454-465.

[16] K.F. Wakker, B.A.C. Ambrosius and L. Aardoom. Precise orbit determination for ERS-1, ESA CR(P)-1842, Delft Univ. Techn., Faculty Aerospace Engin., August 1983.

[17] K.F. Wakker, R.C.A. Zandbergen, G.H.M. van Geldorp and B.A.C. Ambrosius. From satellite altimetry to ocean topography, a survey of data processing techniques, paper presented at EARSeL Symposium on European Remote Sensing Needs in the 1990's, Noordwijkerhout, May 1987.

[18] D.E. Cartwright. Private communication, October 1987.

\section{ACKNOWLEDGEMENT}

The work described in this paper was supported by the Space Research Organization Netherlands (SRON).

We further wish to thank J. Sepers for performing the gravity model tuning experiments. 